\title{
Effect of tyrosol on Staphylococcus aureus antimicrobial susceptibility, biofilm formation and virulence factors
}

\author{
Shaymaa H. Abdel-Rhman and Dina E. Rizk* \\ Department of Microbiology and Immunology, Faculty of Pharmacy, Mansoura University, Mansoura 35516, Egypt.
}

Received 9 March, 2016; Accepted 5 May, 2016

\begin{abstract}
Staphylococcus aureus is considered the most common causative agent of community and hospitalacquired infections. The isolates with increased virulence and antimicrobial resistance, especially mecithillin resistant $S$. aureus (MRSA), represent a challenge in hospitals and health care facilities worldwide. The effect of tyrosol, a phenolic quorum sensing compound of Candida albicans, was studied on the sensitivity of clinical MRSA isolates to antibiotics. Besides having antibacterial activity, subinhibitory concentrations $(3.5-14.3 \mathrm{mM})$ of tyrosol increased the susceptibility to antimicrobial. It gave either synergistic or additive effect when combined with gentamicin, amikacin and ciprofloxacin. Also, the effect of such concentrations on virulence factors production was investigated. Biofilm formed was significantly decreased in most of the tested isolates ( $P \leq 0.0001)$. In addition, it significantly decreased the production of protease and lipase enzymes. Overall, these results represent a promising method for inhibiting $S$. aureus either by reducing its resistance to antibiotics or decreasing the production of virulence factors.
\end{abstract}

Key words: Staphylococcus aureus, tyrosol, antibiotic sensitivity, virulence factors.

\section{INTRODUCTION}

Staphylococcus aureus is a commensal organism that can asymptomatically colonize healthy individuals. Also, it is one of the important pathogens of both hospital and community acquired infections (Gordon and Lowy, 2008; Plata et al., 2009). This pathogen can cause a wide range of infections which may be superfacial, systemic or toxin mediated (Kluytmans et al., 1997; Schito, 2006).

Shortly after the first introduction of methicillin in 1960, methicillin resistant $S$. aureus isolates were reported. MRSA is characterized by the presence of $\operatorname{mec} A$ gene that results in decreased affinity for $\beta$ - lactam antibiotics as it encodes penicillin binding proteins 2A(PBP2A) (Gordon and Lowy, 2008). MRSA is considered a major problem worldwide as it results in worse consequences such as prolonged time of hospitalization, increased cost of treatment and mortality (Onelum et al., 2015). The

*Corresponding author. E-mail: dinaeid2013@yahoo.com or dena@mans.edu.eg. Tel: +201002605440. Fax: 0020502247496.

Author(s) agree that this article remains permanently open access under the terms of the Creative Commons Attribution License 4.0 International License 
increasing prevalence of MRSA requires the development of new treatment strategies for serious infections which became an urgent matter (Shah, 2005).

$S$. aureus possesses an arsenal of virulence determinants that promote tissue colonization, invasion, avoiding the immune systems and dissemination of the disease (Bien et al., 2011). These determinants include adherence factors (adhesins) and secreted products (exoproteins). Adhesins are involved in attachment and initiation of colonization. The organism can reside in biofilm in prosthetic materials causing its persistence and escaping the host defense and antimicrobials (Donlan and Costerton, 2002). Exoproteins such as exotoxins and enzymes (lipases, proteases, hyaluronidase, hemolysins and nucleases) are produced during infection to help in invasion and destruction of tissues (Dinges et al., 2000).

Tyrosol (2-(4-Hydroxyphenyl) ethanol) is a known phenolic compound which is a quorum-sensing molecule of Candida albicans. Similar to farnesol, tyrosol is released into the growth medium and accelerates the formation of germ tubes (Alem et al., 2006). Therefore, it appears that tyrosol and farnesol control positively and negatively the morphogenesis in $C$. albicans.

Moreover, it has antioxidant properties and scavenging effects on reactive oxygen and nitrogen species that are implicated in human pathologies such as cardiovascular and thrombotic diseases. It is found in food sources such as olive oil, olive leaves and green tea (Miro-Casas et al., 2003; Romero and Brenes, 2012) and terrestrial fungi. Tyrosol also showed antimicrobial activity (Alem et al., 2006; Guimarães et al., 2009).

Therefore, this study aims to evaluate the effect of tyrosol on the antimicrobial sensitivity of clinical MRSA isolates, in addition to its effect on biofilm and production of some virulence factors.

\section{MATERIALS AND METHODS}

\section{Bacterial isolates and reagents}

In this study, clinical isolates of $S$. aureus (MRSA) were isolated from Mansoura University Hospitals. MRSA was identified according to sensitivity to cefoxitin disc (CLSI, 2014) and presence of mecA gene by PCR (Kondo et al., 2007). They were stored at $70^{\circ} \mathrm{C}$. Tyrosol and p-nitrophenylpalmitate were purchased from Sigma Chemical Co. (St. Louis, MO, USA). Mueller-Hinton broth (CAMHB, Sigma-Aldrich), LB broth and egg-yolk tellurite emulsion were obtained from Oxoid. MTT (3-(4,5-dimethylthiazol-2-yl)-2,5diphenyltetrazolium bromide) was obtained from SERVA Electrophoresis GmbH (Germany).

\section{Determination of MIC}

The minimal inhibitory concentrations (MICs) of tyrosol, gentamicin, amikacin and ciprofloxacin against $S$. aureus isolates were determined by the broth microdilution method according to CLSI (2014). Briefly, two fold serial dilutions of each tested drug in 96 well microtitreplate were inoculated by cultures of tested isolates. Positive and negative controls were included in each plate. The microtitre plate was incubated at $37^{\circ} \mathrm{C}$, the endpoint was read spectrophotometrically using microplate reader (BioTek ELx800) after $24 \mathrm{~h}$ and the MIC was determined.

\section{Determination of the combined effect of tyrosol and antibiotics}

The combined effect for Tyrosol/gentamicin, tyrosol/amikacin and tyrosol/ciprofloxacin was measured by Checkerboard microdilution method (Orhan et al., 2005). The concentration of each drug ranged from 2 -fold to $1 / 32 \mathrm{MIC}$. One hundred microliter of each antibiotic was combined with $100 \mu$ of tyrosol in 96 well microtitre plates. The plates were inoculated with $20 \mu \mathrm{l}$ of an overnight LB culture giving a final concentration of $5.0 \times 10^{5} \mathrm{CFU} / \mathrm{ml}$ per well. Plates were incubated for $24 \mathrm{~h}$ at $37^{\circ} \mathrm{C}$. To evaluate the interaction, the fractional inhibitory concentration index $(\mathrm{FICl})$ was calculated for each combination according to White et al. (1996) and Abdelmegeed and Shaaban (2013).

\section{Measuring the effect of tyrosol on metabolic activity}

To evaluate the effect of tyrosol on vitality of $S$. aureus isolates, MTT reduction assay was conducted on both tyrosol treated and non-treated cells at a defined time point (Montoro et al., 2005). First, a suspension of an overnight $S$. aureus culture $(100 \mu \mathrm{l})$ was dispensed into selected wells of a 96 -well plate containing tyrosol $(3.5-228.8 \mathrm{mM})$ and incubated for $24 \mathrm{~h}$ at $37^{\circ} \mathrm{C}$. Next, $10 \mu \mathrm{l}$ of MTT solution $\left[5 \mathrm{~g} / \mathrm{l}\right.$ in phosphate buffered saline (PBS) $\left(10 \mathrm{mM} \mathrm{Na}_{2} \mathrm{PO}_{4}\right.$, $1.8 \mathrm{mM} \mathrm{KH}_{2} \mathrm{PO}_{4}, 137 \mathrm{mM} \mathrm{NaCl}, 2.7 \mathrm{mM} \mathrm{KCl}, \mathrm{pH}$ 7.0), filter sterilize by bacterial filter $(0.22 \mu \mathrm{m})$ ] was added to each well and the plate was re-incubated for $4 \mathrm{~h}$. Aspiration of the well solution was performed followed by addition of $50 \mu \mathrm{L}$ of dimethylsulfoxide (DMSO) solution to the wells. The plate was shaken for 5 min. A change in color from yellow to violet was measured with a microtitre plate reader (BioTek ELx800) at $540 \mathrm{~nm}$. All assays were carried out in triplicate.

\section{Biofilm formation}

Biofilm was developed in a 96 well microtiter plate (Nunc, New York, NY, USA). S. aureus cultures $(100 \mu \mathrm{l})$ were incubated for $24 \mathrm{~h}$ at $37^{\circ} \mathrm{C}$ in the presence of different concentrations of tyrosol (3.5$14.3 \mathrm{mM}$ ) and the procedure was completed according to Eid et al. (2012) and El-Mowafy et al. (2014).

\section{Effect of tyrosol on S. aureus virulence factors}

The effect of tyrosol on the production of total protease, lipase and lecithinase enzymes by tested isolates was done with formerly prepared culture supernatant. Total protease activity was measured using the modified skim milk assay as previously described (ElMowafy et al., 2014). The lipase assay was carried out using pnitrophenyl palmitate as a substrate as previously described (ELSokkary et al., 2011).

The lecithinase activity was measured by mixing $100 \mu \mathrm{l}$ of the filtrate with or without tyrosol and $100 \mu \mathrm{l}$ of the egg-yolk suspension (egg-yolk tellurite emulsion $20 \mathrm{ml} / \mathrm{L} ; \mathrm{NaCl}, 5 \mathrm{~g} / \mathrm{L}$ ). The $\mathrm{pH}$ was adjusted to 7.8 in individual wells of polystyrene 96 -well plates then the plates were incubated at $37^{\circ} \mathrm{C}$ for $24 \mathrm{~h}$ and absorbance was measured at $600 \mathrm{~nm}$ using microplate reader. All experiments were carried out in triplicates.

\section{Statistical analysis}

Statistical analysis was done using GraphPad Prism. One way 


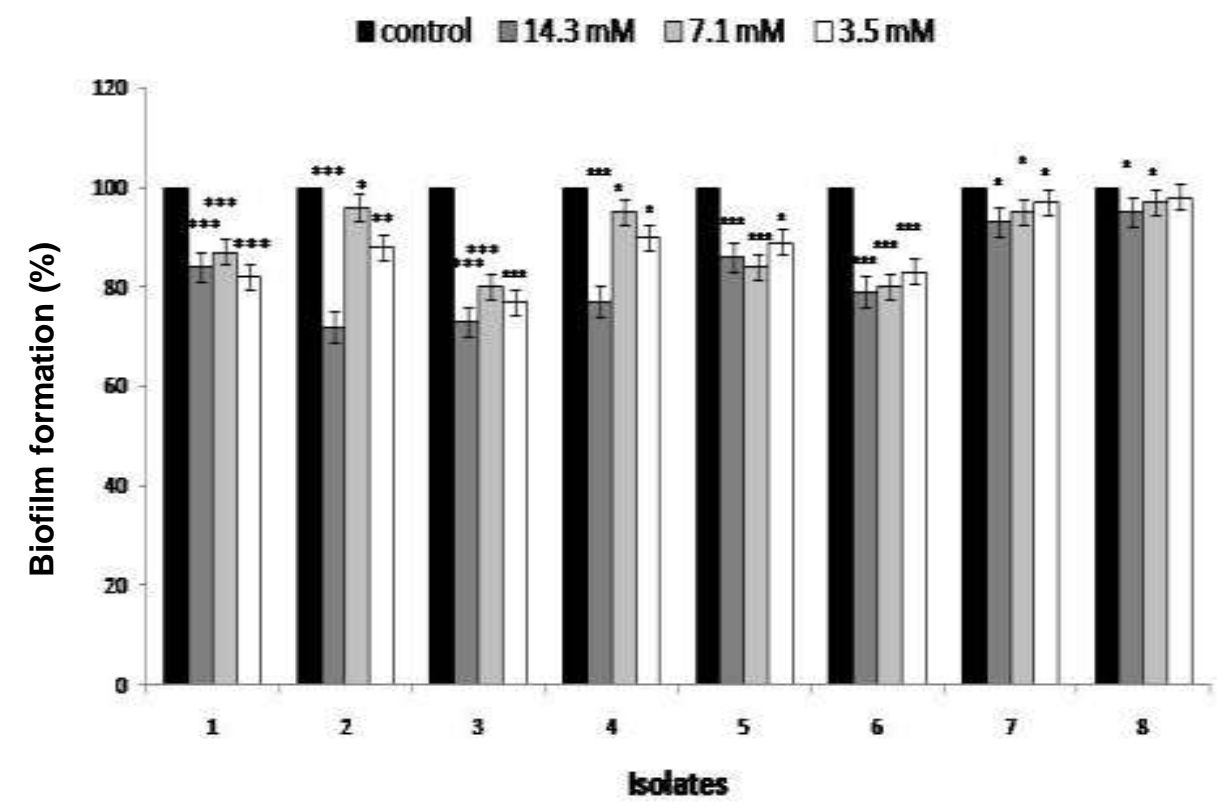

Figure 1. The effect of subinhibitory concentrations (3.5-14.3 mM) of tyrosol on biofilm formation. ( $\left.{ }^{* * *}: \mathrm{P} \leq 0.0001,{ }^{* *}: \mathrm{P} \leq 0.001,{ }^{*}: \mathrm{P} \leq 0.05\right)$.

ANOVA followed by Turky posttest were performed to compare the differences between the control and treated isolates. A P value $\leq$ 0.05 was considered statistically significant.

\section{RESULTS}

\section{MIC determination}

The MIC of tyrosol, gentamicin, amikacin and ciprofloxacin was determined by microbroth dilution method. The MIC of tyrosol was found to be $114.4 \mathrm{mM}$ for all the tested isolates. The MIC for gentamicin, amikacin and ciprofloxacin were $62.5-8000,17.5-70$ and $125-500$ $\mu \mathrm{g} / \mathrm{ml}$, respectively.

\section{The combined effect of tyrosol and antibiotics}

The effect of tyrosol/gentamicin combination was found to be synergistic in $66 \%$ of tested isolates ( $\mathrm{FICl}=0.28-0.5)$. Besides that, tyrosol/amikacin and tyrosol/ciprofloxacin combinations exhibited synergistic effect in two isolates $(\mathrm{FICl}=0.37)$. For the other isolates, these three combinations showed an additive effect ( $\mathrm{FICl}=0.51-0.62)$.

\section{Effect of tyrosol on the metabolic activity}

MTT, an oxidation-reduction indicator was added to suspensions in the presence of tyrosol. The vitality assay indicated that incubation of cells with 114.4 and 228.8
$\mathrm{mM}$ of tyrosol (1x and $2 \mathrm{x} \mathrm{MIC}$ ) resulted in no change in color from yellow to violet, showing that these concentrations prevented the oxidation-reduction reactions in the suspensions. Tyrosol (28.6- $57.2 \mathrm{mM}$ ) partially inhibited oxidation-reduction reactions. Lower concentrations (3.5 $14.3 \mathrm{mM}$ ) had no effect on the viability of tested isolates. Therefore, these subinhibitory concentrations were used for studying the effect of tyrosol on the production of virulence factors.

\section{Biofilm formation}

The effect of $3.5-14.3 \mathrm{mM}$ of tyrosol on biofilm formation was estimated. All the tested concentrations reduced the formed biofilm of the isolates significantly except for one isolate in which $(3.5 \mathrm{mM})$ tyrosol reduced the formed biofilm in non-significant manner (Figure 1). The maximum decrease $(30 \%)$ in biofilm formation was attained using $3.5 \mathrm{mM}$ of tyrosol among isolates 2, 3 and 4.

\section{Effect of tyrosol on virulence factors}

Sub-inhibitory concentrations $(3.5-14.3 \mathrm{mM})$ of tyrosol were found to significantly decrease protease and lipase enzyme production in MRSA isolates 3,5 and 7. Tyrosol (14.3 $\mathrm{mM}$ ) significantly decreased protease and lipase release with $\mathrm{P}<0.05$ by $35-40 \%$ (Figure 2 ) and $25-40 \%$ (Figure 3), respectively. On the other hand, lecithinase enzyme production was increased significantly in all the 


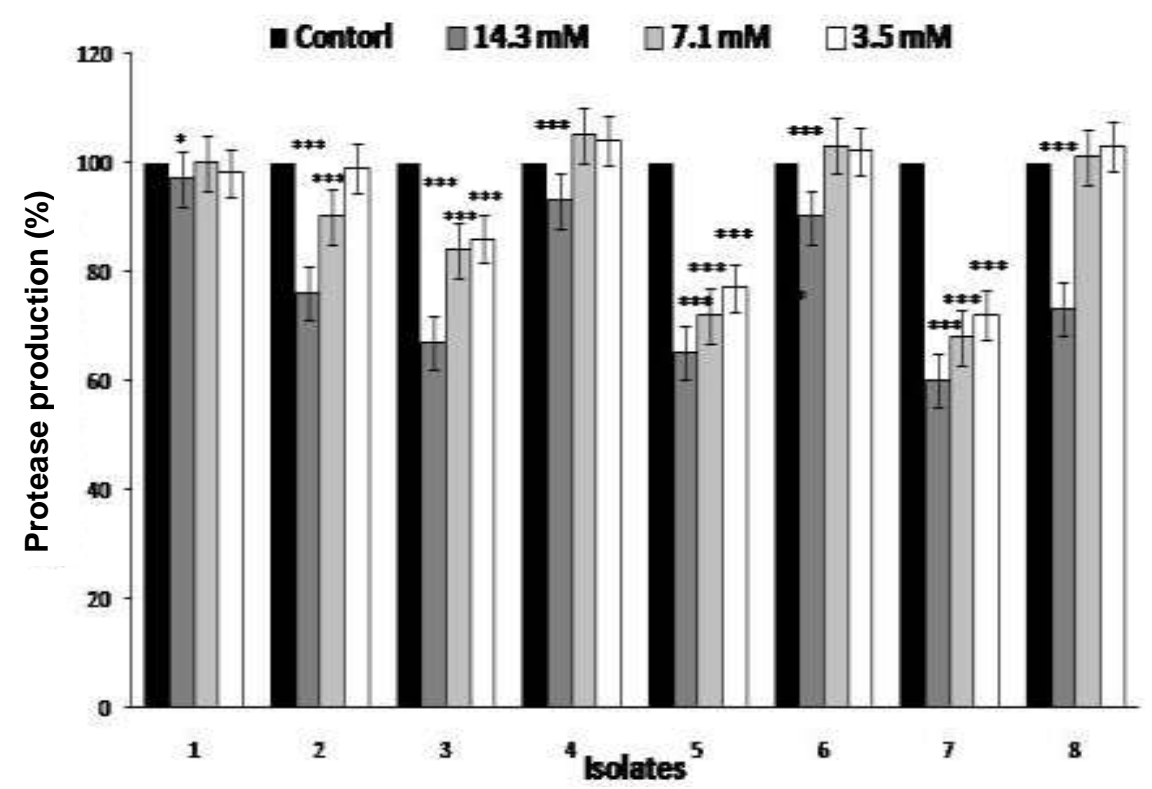

Figure 2. The effect of subinhibitory concentrations (3.5-14.3 mM) of tyrosol on protease enzyme production. ( ${ }^{* * *} ; \mathrm{P} \leq 0.0001,{ }^{* *}$; $\mathrm{P} \leq 0.001$, *; $\mathrm{P} \leq 0.05$ )

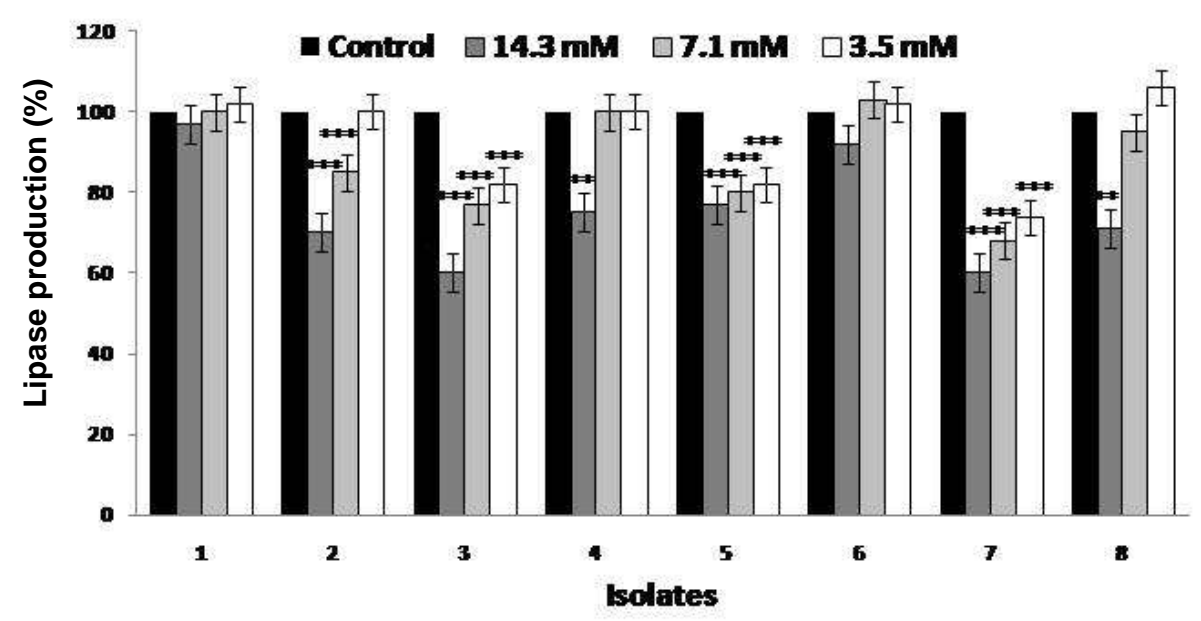

Figure 3. The effect of subinhibitory concentrations (3.5-14.3 mM) of tyrosol on lipase enzyme production. $\left({ }^{* * *}: \mathrm{P} \leq 0.0001,{ }^{* *}: \mathrm{P} \leq 0.001,{ }^{*}\right.$ : $\left.\mathrm{P} \leq 0.05\right)$.

tested isolates except for one isolate in which $3.5 \mathrm{mM}$ of tyrosol led to non-significant increase (Figure 4).

\section{DISCUSSION}

There is mounting indication in the literature for the prominence of polymicrobial infections in which organisms interact with each other synergistically or inhibiting the effect of their pathogenesis and patient health (Harriott and Noverr, 2009). C. albicans cohabits with various human microbiota in multiple sites which give rise to mixed species biofilm (Shirtliff et al., 2009; Peleg et al., 2010; Ovchinnikova et al., 2012). In poly- microbial blood stream infections, C. albicans was co-isolated with $S$. aureus in 11\% of cases (Klotz et al., 2007).

Besides that, $C$. albicans and $S$. aureus can form complex polymicrobial biofilms on various mucosal surfaces in which $S$. aureus is associated with hyphal cells (Peters et al., 2010). The effect of $C$. albians itself on $S$. aureus has been extensively investigated previously (Harriott and Noverr, 2009; Ovchinnikova et 


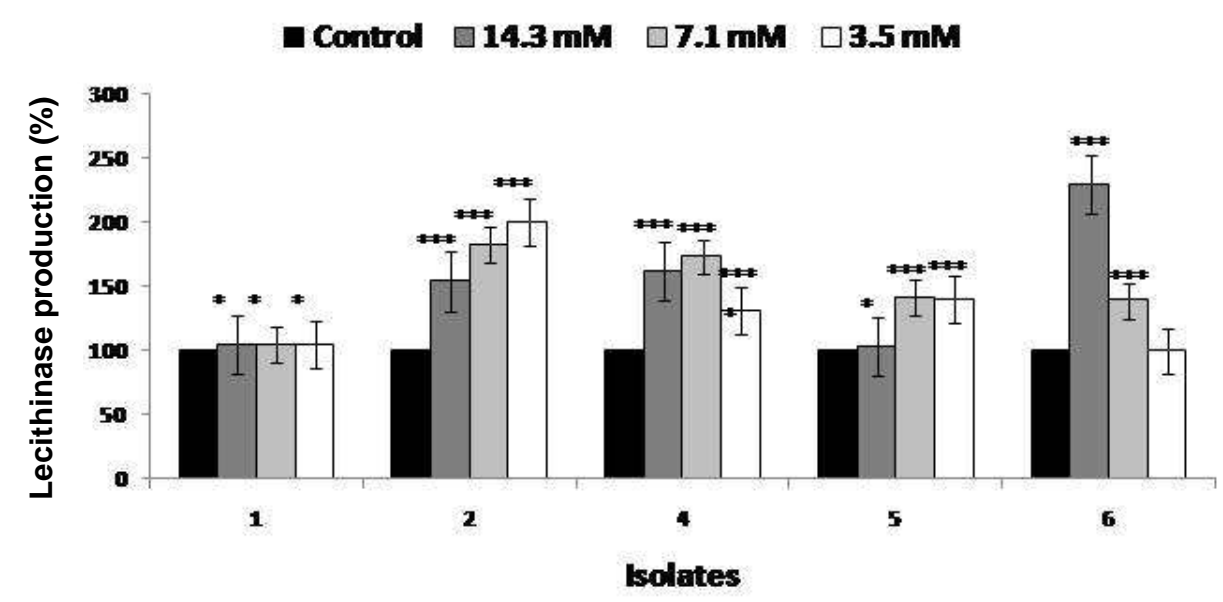

Figure 4. The effect of subinhibitory concentrations (3.5-14.3 $\mathrm{mM})$ of tyrosol on lecithinase enzyme production. ( ${ }^{* * *}: \mathrm{P} \leq 0.0001$, ${ }^{* *}$ : $\mathrm{P} \leq 0.001$, *: $\left.\mathrm{P} \leq 0.05\right)$.

al., 2012; Lin et al., 2013; Schlecht et al., 2015). The effect of farnesol, $C$. albicans quorum sensing compound, was studied on $S$. aureus in various reports (Unnanuntana et al., 2009; Kaneko et al., 2011; Cerca et al., 2013). Till now, no available investigation was done to evaluate the effect of the other $C$. albicans quorum sensing compound (tyrosol) on the sensitivity of $S$. aureus to antibiotics and virulence production.

In this study, the authors were interested in determining how tyrosol might affect the antibiotic sensitivity, biofilm and some virulence factors production (protease, lipase and lecithinase) by Egyptian clinical isolates of $S$. aureus.

Tyrosol exhibits antibacterial effect against $S$. aureus. In contrast to these results, Papadopoulou et al. (2005) showed that tyrosol is least likely to exhibit any antimicrobial activity on $S$. aureus, E. coli and C. albicans. Being a phenolic compound may account for this antibacterial activity by having membrane-active properties which induce progressive leakage of intracellular constituents, including $\mathrm{K}^{+}$(Lambert and Hammond, 1973). Aside from being a quorum sensing compound, tyrosol is one of the simple phenols found in olive oil providing benefits to health. Medina et al. (2006) reported that hydroxytyrosol and tyrosol are olive oil phenolic compounds having bactericidal activity against the tested foodborne pathogens including $S$. aureus.

The ability of tyrosol to sensitize the tested isolates to antibiotics (gentamicin, amikacin and ciprofloxacin) was assessed. It enhanced the activity of the three antibiotics against $S$. aureus isolates. About $66.7 \%$ of tested isolates exhibited sensitivity towards the tyrosol/gentamicin combination. For amikacin and ciprofloxacin, tyrosol exhibited a synergistic effect in two out of eight isolates and showed additive effect in the other isolates. The synergistic effect shown by tyrosol/ antibiotic combinations may be explained by the possible membrane permealizing action of tyrosol which increases the sensitivity of $S$. aureus to antibiotics. A similar finding was reported by Jabra-Rizk et al. (2006) who showed that farnesol increases the sensitivity of tested mecithillin sensitive $S$. aureus isolates to gentamicin and this increase was not shown in MRSA isolates. Unnanuntana et al. (2009) have demonstrated that farnesol/gentamicin combination has no effect on the sensitivity of $S$. aureus to gentamicin. Concerning farnesol, it has variable activities against different organisms in combination with different antimicrobials. For example, a study performed by Abdel-Rhman et al. (2015) showed that neither farnesol nor tyrosol at their subinhibitory concentrations had any effect on the activity of antibiotics on Pseudomonas aeruginosa.

Bacterial biofilm is a microbial derived sessile community, where cells are embedded in extracellular polymeric matrix. Cells in biofilm show an altered phenotype in growth, gene expression and protein production. Biofilm formation requires first adherence of organisms to surfaces, followed by proliferation and finally biofilm formation (Jabra-Rizk et al., 2006; Unnanuntana et al., 2009). In the current study, subinhibitory concentrations of tyrosol reduced biofilm formation significantly. This result is consistent with that of Arias et al. (2016) who demonstrated that tyrosol reduces single and mixed biofilms formed by $C$. albicans and Streptococcus mutans. Also, Jabra-Rizk et al. (2006) found that farnesol has the ability to inhibit biofilm formation by $S$. aureus.

Moreover, the effect of tyrosol on the production of some virulence factors of $S$. aureus was studied. Tyrosol subinhibitory concentrations $(3.5-14.3 \mathrm{mM})$ reduced protease enzyme production in 100,50 and $37.5 \%$ of the tested isolates, respectively. This result coincides with that of Abdel-Rhman et al. (2015) who reported a similar result against $P$. aeruginosa.

Another virulence factor of $S$. aureus is lipase that 
contributes to skin colonization through hydrolyzation of human sebum. Moreover, the effect of tyrosol on lipase production was investigated. Tyrosol $(14.3 \mathrm{mM})$ caused $25-40 \%$ reduction of lipase production in six isolates, 7.1 $\mathrm{mM}$ resulted in 20-30\% reduction in four isolates and 3.5 $\mathrm{mM}$ resulted in about $20 \%$ reduction in only three isolates. These results are consistent with what reported by Kuroda et al. (2007) who found that subinhibitory MIC of farnesol inhibited lipase activity in $S$. aureus. On the contrary, lecithinase was significantly increased by the tested concentrations of tyrosol. None of the previous studies have investigated the effect of either tyrosol or farnesol on lecithinase production. So, this result requires further study to explain this increase by studying its effect on agr (accessory gene regulator) quorum-sensing system of $S$. aureus which controls the expression of many secreted virulence factors.

\section{Conclusion}

The present study results demonstrate the beneficial effects of tyrosol in relation to antimicrobial susceptibility and virulence factors production of $S$. aureus. This sheds more light on the therapeutic use of tyrosol against $S$. aureus infections. However, more studies are required to reveal its effect on quorum sensing system that controls virulence gene expression.

\section{Conflict of Interests}

The authors have not declared any conflict of interest.

\section{REFERENCES}

Abdel-Rhman SH, Mostafa El-Mahdy A, El-Mowafy M (2015). Effect of Tyrosol and Farnesol on Virulence and Antibiotic Resistance of Clinical Isolates of Pseudomonas aeruginosa. Biomed. Res. Int. 2015:456463.

Abdelmegeed E, Shaaban MI (2013). Cyclooxygenase inhibitors reduce biofilm formation and yeast-hypha conversion of fluconazole resistant Candida albicans. J. Microbiol. 51(5):598-604.

Alem MA, Oteef MD, Flowers TH, Douglas LJ (2006). Production of tyrosol by Candida albicans biofilms and its role in quorum sensing and biofilm development. Eukaryot. Cell 5(10): 1770-1779.

Arias LS, Delbem AC, Fernandes RA, Barbosa DB, Monteiro DR (2016). Activity of tyrosol against single and mixed-species oral biofilms. J. Appl. Microbiol. 120(5):1240-1249.

Bien J, Sokolova O, Bozko P (2011). Characterization of virulence factors of Staphylococcus aureus: Novel function of known virulence factors that are implicated in activation of airway epithelial proinflammatory response. J. Pathog. 2011:601905.

Cerca N, Gomes F, Bento JC, Franca A, Rolo J, Miragaia M, Teixeira P, Oliveira R (2013). Farnesol induces cell detachment from established S. epidermidis biofilms. J. Antibiot. 66(5): 255-258.

CLSI (2014). Performance standards for antimicrobial susceptibility testing: Twenty-fourth informational supplement. Clincal and laboratory standards institute Wayne.PA. M100-S24.

Dinges MM, Orwin PM, Schlievert PM (2000). Exotoxins of Staphylococcus aureus. Clin. Microbiol. Rev. 13(1): 16-34. PMID 88931
Donlan RM, Costerton JW (2002). Biofilms: Survival mechanisms of clinically relevant microorganisms. Clin. Microbiol. Rev. 15(2):167193.

Eid D, Naggar WE, Barwa R, El-Sokkary MA (2012). Phenotypic and genotypic characterization of some virulence factors in Pseudomonas aeruginosa isolates isolated from different clinical sources in Mansoura University Hospitals. N. Egypt. J. Microbiol. 32(2):151-167.

El-Mowafy SA, Abd El Galil KH, El-Messery SM and Shaaban MI (2014). Aspirin is an efficient inhibitor of quorum sensing, virulence and toxins in Pseudomonas aeruginosa. Microb. Pathog. 74: 25-32.

EL-Sokkary MA, E.Habib E, Shima H (2011). Production of lipase enzyme by clinical Pseudomans species isolated from Mansoura University hospitals. Egypt. J. Biotechnol. 38: 1-15.

Gordon RJ, Lowy FD (2008). Pathogenesis of methicillin-resistant Staphylococcus aureus infection. Clin. Infect. Dis. 46 Suppl 5: S350359.

Guimarães DO, BorgesII KB, Bonatoll PS, Pupo MT (2009). A simple method for the quantitative analysis of tyrosol by HPLC in liquid Czapek cultures from endophytic fungi. J. Braz. Chem. Soc. 20(1).

Harriott MM, Noverr MC (2009). Candida albicans and Staphylococcus aureus form polymicrobial biofilms: Effects on antimicrobial resistance. Antimicrob. Agents Chemother. 53(9): 3914-3922.

Jabra-Rizk MA, Meiller TF, James CE, Shirtliff ME (2006). Effect of farnesol on Staphylococcus aureus biofilm formation and antimicrobial susceptibility. Antimicrob. Agents Chemother. 50(4): 1463-1469.

Kaneko M, Togashi N, Hamashima H, Hirohara M, Inoue Y (2011). Effect of farnesol on mevalonate pathway of Staphylococcus aureus. J. Antibiot. 64(8):547-549.

Klotz SA, Chasin BS, Powell B, Gaur NK, Lipke PN (2007). Polymicrobial bloodstream infections involving Candida species: Analysis of patients and review of the literature. Diagn. Microbiol. Infect. Dis. 59(4):401-406.

Kluytmans J, van Belkum A, Verbrugh H (1997). Nasal carriage of Staphylococcus aureus: Epidemiology, underlying mechanisms, and associated risks. Clin. Microbiol. Rev. 10(3):505-520.

Kondo $\mathrm{Y}$, Ito $\mathrm{T}, \mathrm{Ma} \mathrm{XX}$, Watanabe $\mathrm{S}$, Kreiswirth BN, Etienne J, Hiramatsu K (2007). Combination of multiplex PCRs for staphylococcal cassette chromosome mec type assignment: Rapid identification system for $\mathrm{mec}, \mathrm{ccr}$, and major differences in junkyard regions. Antimicrob. Agents Chemother. 51(1):264-274.

Kuroda M, Nagasaki S, Ito R, Ohta T (2007). Sesquiterpene farnesol as a competitive inhibitor of lipase activity of Staphylococcus aureus. FEMS Microbiol. Lett. 273(1):28-34.

Lambert PA, Hammond SM (1973). Potassium fluxes, first indications of membrane damage in micro-organisms. Biochem. Biophs. Res. Commun. 54(2):796-799.

Lin YJ, Alsad L, Vogel F, Koppar S, Nevarez L, Auguste F, Seymour J, Syed A, Christoph K, Loomis JS (2013). Interactions between Candida albicans and Staphylococcus aureus within mixed species biofilms. Bios. 84(1):30-39.

Medina E, de Castro A, Romero C, Brenes M (2006). Comparison of the concentrations of phenolic compounds in olive oils and other plant oils: Correlation with antimicrobial activity. J. Agric. Food Chem. 54(14):4954-4961.

Miro-Casas E, Covas MI, Fito M, Farre-Albadalejo M, Marrugat $\mathrm{J}$, de la Torre R (2003). Tyrosol and hydroxytyrosol are absorbed from moderate and sustained doses of virgin olive oil in humans. Eur. J. Clin. Nutr. 57(1): 186-190.

Montoro E, Lemus D, Echemendia M, Martin A, Portaels F, Palomino JC (2005). Comparative evaluation of the nitrate reduction assay, the MTT test, and the resazurin microtitre assay for drug susceptibility testing of clinical isolates of Mycobacterium tuberculosis. $\mathrm{J}$. Antimicrob. Chemother. 55(4):500-505.

Onelum O, Odetoyin B, Onipede A, Oyelese A (2015). The role of methicillin-resistant Staphylococcus aureus in clinical infections in Obafemi Awolowo University Teaching Hospitals Complex, Ile-lfe, South Western Nigeria. J. Microbiol. Exp. 2(2): 00041.

Orhan G, Bayram A, Zer Y, Balci I (2005). Synergy tests by E test and checkerboard methods of antimicrobial combinations against Brucella melitensis. J. Clin. Microbiol. 43(1):140-143.

Ovchinnikova ES, Krom BP, Busscher HJ, van der Mei HC (2012). 
Evaluation of adhesion forces of Staphylococcus aureus along the length of Candida albicans hyphae. BMC Microbiol. 12:281.

Papadopoulou C, Soulti K, Roussis IG (2005). Potential antimicrobial activity of red and white wine phenolic extracts against isolates of Staphylococcus aureus, Escherichia coli and Candida albicans. Food Technol. Biotechnol. 43(1):41-46.

Peleg AY, Hogan DA, Mylonakis E (2010). Medically important bacterial-fungal interactions. Nat. Rev. Microbiol 8(5):340-349.

Peters BM, Jabra-Rizk MA, Scheper MA, Leid JG, Costerton JW, Shirtliff ME (2010). Microbial interactions and differential protein expression in Staphylococcus aureus -Candida albicans dual-species biofilms. FEMS Immunol. Med. Microbiol. 59(3):493-503.

Plata K, Rosato AE, Wegrzyn G (2009). Staphylococcus aureus as an infectious agent: Overview of biochemistry and molecular genetics of its pathogenicity. Acta Biochem. Pol. 56(4): 597-612.

Romero C, Brenes M (2012). Analysis of total contents of hydroxytyrosol and tyrosol in olive oils. J. Agric. Food Chem. 60(36):9017-9022.

Schito GC (2006). The importance of the development of antibiotic resistance in Staphylococcus aureus. Clin. Microbiol. Infect. 12 Suppl 1:3-8.

Schlecht LM, Peters BM, Krom BP, Freiberg JA, Hansch GM, Filler SG, Jabra-Rizk MA, Shirtliff ME (2015). Systemic Staphylococcus aureus infection mediated by Candida albicans hyphal invasion of mucosal tissue. Microbiology 161(Pt 1):168-181.
Shah PM (2005). The need for new therapeutic agents: What is the pipeline? Clin. Microbiol. Infect. 11(Suppl. 3):36-42.

Shirtliff ME, Peters BM, Jabra-Rizk MA (2009). Cross-kingdom interactions: Candida albicans and bacteria. FEMS Microbiol. Lett. 299(1):1-8.

Unnanuntana A, Bonsignore L, Shirtliff ME, Greenfield EM (2009). The effects of farnesol on Staphylococcus aureus biofilms and osteoblasts. An in vitro study. J. Bone Joint Surg. Am. 91(11):26832692.

White RL, Burgess DS, Manduru M, Bosso JA (1996). Comparison of three different in vitro methods of detecting synergy: Time-kill, checkerboard, and $\mathrm{E}$ test. Antimicrob. Agents Chemother. 40(8):1914-1918. 